\title{
The role of miRNAs in human papilloma virus (HPV)-associated cancers: bridging between HPV-related head and neck cancer and cervical cancer
}

\section{CB Lajer ${ }^{*, 1,8}$, E Garnæs ${ }^{1,8}$, L Friis-Hansen ${ }^{2}$, B Norrild ${ }^{3}$, MH Therkildsen ${ }^{4}$, M Glud $^{2}$, M Rossing $^{4}$, H Lajer $^{5}$, D Svane ${ }^{5}$, L Skotte ${ }^{6}$, L Specht ${ }^{7}$, C Buchwald' and FC Nielsen ${ }^{2}$}

'Department of Otorhinolaryngology, Head and Neck Surgery and Audiology, Rigshospitalet, University of Copenhagen, 2 I 00 Copenhagen, Denmark; ${ }^{2}$ Department of Clinical Biochemistry, Rigshospitalet, University of Copenhagen, 2100 Copenhagen, Denmark; ${ }^{3}$ Department of Cellular and Molecular Medicine, Panum Institute, University of Copenhagen, 2200 Copenhagen, Denmark; ${ }^{4}$ Department of Pathology, Rigshospitalet, University of Copenhagen, 2100 Copenhagen, Denmark; ${ }^{5}$ Department of Gynaecology and Obstetrics, Rigshospitalet, University of Copenhagen, 2100 Copenhagen, Denmark; ${ }^{6}$ Department of Biology, Bioinformatics Centre, University of Copenhagen, 2200 Copenhagen, Denmark; 'Department of Oncology, Rigshospitalet, University of Copenhagen, 2100 Copenhagen, Denmark

BACKGROUND: Although the role of human papilloma virus (HPV) in cervical squamous cell carcinoma (CSCC) is well established, the role in head and neck SCC (HNSCC) is less clear. MicroRNAs (miRNAs) have a role in the cancer development, and HPV status may affect the miRNA expression pattern in HNSCC. To explore the influence of HPV in HNSCC, we made a comparative miRNA profile of HPV-positive (HPV +) and HPV-negative (HPV - ) HNSCC against CSCC.

METHODS: Fresh frozen and laser microdissected-paraffin-embedded samples obtained from patients with HPV + /HPV - HNSCC, CSCC and controls were used for microarray analysis. Differentially expressed miRNAs in the HPV + and HPV - HNSCC samples were compared with the differentially expressed miRNAs in the CSCC samples.

RESULTS: Human papilloma virus positive $(+)$ HNSCC had a distinct miRNA profile compared with HPV - HNSCC. Significantly more similarity was seen between HPV + HNSCC and CSCC than HPV - and CSCC. A set of HPV core miRNAs were identified. Of these especially the miR-I5a/miR-I6/miRI95/miR-497 family, miR-I43/miR-I45 and the miR-106-363 cluster appear to be important within the known HPV pathogenesis.

CONCLUSION: This study adds new knowledge to the known pathogenic pathways of HPV and substantiates the oncogenic role of HPV in subsets of HNSCCs.

British Journal of Cancer (2012) I 06, I526-I534. doi:I0.1038/bjc.20 I2.109 www.bjcancer.com

Published online 3 April 2012

(c) 2012 Cancer Research UK

Keywords: microRNA; human papilloma virus; cervical cancer; head and neck cancer; tonsillar cancer

Head and neck cancers are the sixth most common cancer in the world (Warnakulasuriya, 2009). They consist of a heterogeneous group of neoplasms, derived from the epithelium in the upper aerodigestive tract. The most common type is squamous cell carcinoma (SCC) occurring in the oral cavity, pharynx or larynx. Tobacco and alcohol are the predominant risk factors in head and neck SCC (HNSCC), but human papilloma virus (HPV) is also established to be associated with HNSCC, especially in the case of oropharyngeal SCC, that is, in the tonsils and base of the tongue. Although the incidence of tobacco- and alcoholinduced cancers is declining, there are solid, indications that the incidence as well as the prevalence of HPV-induced HNSCCs is increasing (Nasman et al, 2009). In Denmark, the incidence of oropharyngeal cancers has doubled from 1999 to 2008, in both men and women (the Danish Cancer Registry http://www.sst.dk).

*Correspondence: Dr CB Lajer; E-mail: christel.lajer@rh.regionh.dk

${ }^{8}$ Shared first authorship.

Received 25 January 2012; revised 7 March 2012; accepted 7 March 2012; published online 3 April 2012
Human papilloma virus + HNSCC is distinct from HPV-negative (HPV - ) HNSCC in a number of ways. Patients with HPV + HNSCC tend to be younger and have a lower intake of tobacco and alcohol. Moreover, HPV + tumours are usually smaller in $\mathrm{T}$-size but with large cystic nodal involvement $(\mathrm{N}+)$. The tumours are frequently non-keratinised (Lajer and Buchwald, 2010), but normally have favourable prognosis compared with HPV - HNSCC (Hafkamp et al, 2008; Lassen et al, 2009). Today, it is well established that particular types of HPV cause cervical and anogenital cancer. More than $99 \%$ of cervical cancer biopsies contain high-risk HPV genomes A (Walboomers et al, 1999), and cervical cancer is preceded by precancerous lesions, which are effectively prevented by vaccination against HPV (Majewski et al, 2009). The correlation between HNSCC and HPV is less clear. Even though HPV + cancers are recognised as a distinct subgroup within HNSCC (Syrjanen et al, 1983a, b), precancerous lesions are not recognised, in oropharyngeal cancers, and the role of HPV in HNSCC is still not fully established. The issue has major implications for the clinical handling of HNSCC, as a causal function of HPV would implicate, that HPV vaccines 
could be effective in preventing HNSCC in the same way as cervical cancers.

One way to expand the understanding of HPV in HNSCC is to define the molecular perturbations in HPV + tumours and compare them to those occurring in cervical cancers.

The course of malignant transformation in cells infected with oncogenic HPV types is incompletely understood, but some components are known (Wise-Draper and Wells, 2008). Virally encoded E6 binds simultaneously to the ubiquitin/protein ligase E6AP and p53, resulting in ubiquitination of p53 and its subsequent proteolytic degradation. Human papilloma virus E7 protein moreover binds and destabilises retinoblastoma protein $(\mathrm{pRb})$ and reduces binding to E2F and thereby promotes cell cycle progression. Inactivation of $\mathrm{pRb}$ also results in a reciprocal overexpression of p16 tumour suppressor protein p16INK4A, which inhibits phosphorylation of the Rb-E2F complex (Ghittoni et al, 2010;Pim and Banks, 2010).

Among the large number of different biomarkers microRNAs (miRNAs) have previously been reported to be intimately connected to cellular transformation. microRNAs are small 21-22n non-protein-coding RNAs that regulate mRNA translation and decay. microRNAs are involved in terminal differentiation processes and have a significant role in the development of human cancer. microRNA patterns are tissue-specific and it is normally possible to distinguish carcinomas from normal cells. At the same time miRNAs are potential therapeutic targets or therapeutic agents in future cancer treatments (Pan et al, 2010). We only have limited information about miRNAs in HPV + HNSCC cancer. Wald et al (2011) recently examined cultured HPV + and HPV SCC cells and identified a number of perturbed miRNAs in the $\mathrm{HPV}+$ cells. The expression of the HPV-16 E6 oncogene was sufficient to alter miRNA expression (Wald et al, 2011), indicating that HPV was directly connected to the observed changes. In agreement with these observations, we previously reported a difference in the miRNA profiles between the HPV + and HPV pharyngeal SCCs (PSCCs; Lajer et al, 2011).

To further characterise these differences, we expand our previous observations by applying laser microdissection on tonsillar SCC (TSCC) to isolate cancerous and non-cancerous tissue and compare the miRNA signatures from $\mathrm{HPV}+$ and HPV - tumours with those of CSCCs. The aim of this study is to substantiate the distinct oncogenic role of HPV in a subset of HNSCC by showing that the miRNA expression in HPV + and HPV - HNSCC is significantly different and that miRNA profiles in HPV + HNSCC show similarities with HPV + CSCC. By making a link to CSCC, we aim to explore the common virus-induced molecular changes in the cell that leads to the malignant state.

\section{MATERIALS AND METHODS}

\section{Patients and tumour samples}

All patients with pharyngeal cancer and controls (fresh frozen samples as well as formalin-fixed paraffin-embedded (FFPE) samples) in this study had surgery performed at the Department of Oto-rhino-laryngology, Head \& Neck Surgery and Audiology, Rigshospitalet, University of Copenhagen. The department is responsible for the surgical treatment of head and neck cancer patients in East Denmark, the Faeroe Islands and Greenland and receives patients from all of Denmark for treatment at highly specialised level. Cervical SCC patients and controls had primary surgery done at the Department of Gynaecology, Rigshospitalet. All fresh frozen samples were taken during primary surgery from vital tumour tissue. Patients were included at the consultation primary to surgery.

The study was approved by the Regional Scientific Ethical Committee (H-C-2008-080).

\section{Paraffin-embedded tonsillar samples}

Formalin-fixed paraffin-embedded tonsillar cancers from the period 2008-2010 were collected and p16 immunohistochemistry was performed on all samples. On the basis of the amount of tumour tissue in the sections, 31 tumour samples were selected for laser microdissection and 13 samples from patients with nonneoplastic tonsillar disease were included as controls. The latter were typically derived from tonsillectomies performed on chronic tonsillitis patients. All specimens were reviewed by a head and neck pathologist to ensure the pathological diagnosis.

\section{Fresh frozen oral cavity and pharyngeal cancer}

Fresh frozen tumour samples from the oral cavity and pharynx is previously described in Lajer et al (2011). In the previous study, PSCC samples had some degree of heterogeneity, probably because of the presence of normal tissue in the PSCC biopsies. In this study, we therefore included only samples from large tumours that were primarily excised and thereby excluded four samples that were suspected to contain a high degree of normal tissue.

\section{Fresh frozen cervical samples}

All cervical cancer patients provided informed consent for the use of tissue harvested at surgery.

A total of 16 patients with cervical cancer and 16 patients with non-neoplastic diseases in the uterus had biopsies taken during surgery. The biopsies were placed directly in RNA-later and frozen within an hour. Samples from $10 \mathrm{HPV}+$ CSCC patients and 10 $\mathrm{HPV}$ - control patients were used for miRNA profiling. Five of the originally 16 cervical cancer samples were adenocarcinomas and 1 patient sample was very small and these were excluded for the miRNA profiling. One control sample was tested positive for HPV. This sample and additionally 5 other control samples were likewise excluded to obtain the same number of cancer and control samples. Information of diagnosis, smoking and alcohol abuse, differentiation grade and TNM stage were collected from the patient's charts.

\section{Microdissection}

The laser microdissection was performed on a PALM MicroBeam (Carl Zeiss Microimaging GmbH, Jena, Germany). Each tissue block was cut according to the standard protocol into $10 \mu \mathrm{m}$ sections and placed on MMI RNAse-free frame PEN membrane slides. Sections were deparaffinised and stained with cresyl violet using the Ambions LCM staining kit made for RNA isolation (Ambion, Life Technologies Corporation, Carlsbad, CA, USA). Our cutoff level in cancer sections was achieving $\geqslant 80 \%$ tumour cells in the dissected areas. In the control samples, tissue areas of stratified squamous cell epithelium were excised. Tubes were immediately frozen at $-80{ }^{\circ} \mathrm{C}$ until further processing. During microdissection one to four pictures were taken of each cancer and controls for documentation (Supplementary Figure 1).

\section{RNA extraction}

From fresh frozen biopsies, total RNA was extracted and isolated by homogenising the frozen samples in TRIzol (Invitrogen, Life Technologies Corporation, Carlsbad, CA, USA) using a homogeniser (Tissuelyser, Qiagen GmbH, BY, Germany). Briefly, the total RNA was extracted from tissue lysate by phase separation with 1-Bromo-3chloropropane and RNA precipitation with isopropanol. After washing with alcohol the RNA was eluted in RNAse-free water. Total RNA concentration of samples was measured using a Nanodrop ND1000 Spectrophotometer (Wilmington, DE, USA).

In the case of FFPE specimens RNA was isolated using a combination of Ambions Recover All and Ambions RNAqueous. 


\section{DNA extraction}

DNA from fresh frozen biopsies was extracted from the interphase of the Trizol and precipitated with ethanol. It was dissolved in lysate buffer and proteinase $\mathrm{K}$, and after a second precipitation with ethanol, the solutions were transferred to spin columns. After washing, DNA was eluted with water. DNA from FFPE specimens was isolated from four $10 \mu \mathrm{m}$ sections using the Qiagens QIAamp DNA FFPE Tissue Kit to spin columns (NucleoSpin Tissue).

\section{HPV testing}

Immunohistochemistry for $\mathrm{p} 16^{\mathrm{INK} 4 \mathrm{~A}}$ was performed on FFPE tissue slides pre-treated in DAKO (Dako Denmark A/S, Glostrup, Denmark) PT Link, incubated with p16 (JC8) antibody, Santa Cruz, (Santa Cruz Biotechnology Inc., Santa Cruz, CA, USA) and visualised using the DAKO Envision Flex + system. Slides were categorised as positive, if there was a strong diffuse nuclear and cytoplasmic staining of $>50 \%$ of the tumour cells. PCR for HPV was performed with general primers MY09 and MY11 and GP5 + I GP6 + . Validation of the DNA quality and the efficacy of the PCR were done with PCR for the house keeping gene GAPDH. Tissue with a positive GAPDH PCR was considered HPV + by PCR if either one or two positive primer sets were positive. Cancers were classified as HPV + based on both positive PCR and positive p16 staining. Cancers with a negative PCR were considered HPVwithout regards of the p16 immunohistochemistry. None of the PCR + samples were p16 negative. According to these preliminary investigations, $10 \mathrm{HPV}+$ and $10 \mathrm{HPV}$ - cancers were selected for laser microdissection. The 10 control tonsils were selected for microdissection based on a positive GAPDH PCR. Nine controls were $\mathrm{HPV}-$ and one was HPV + . All HPV + samples were additionally tested positive with HPV-type 16- and -type 11specific primers. All HPV+ PSCC and FFPE TSCC were positive for the type 16-specific primers and additionally negative for type 11 -specific primers. The $10 \mathrm{HPV}+\mathrm{CSCC}$ samples were typed for HPV-type 16 and 18. Seven samples were HPV-type 16 positive and three were type 18 positive.

\section{MiRNA array and data deduction}

A $500 \mathrm{ng}$ of total RNA was labelled with FlashTag Biotin RNA Labelling Kits from Genisphere (Genisphere LLC, Hatfield, PA, USA) and hybridised to Affymetrix GeneChip miRNA Array according to the manufacturer's instructions (Affymetrix, Santa Clara, CA, USA).

The Affymetrix miRNA Array comprise probes for 847 human miRNAs. Four copies of each miRNA probe are distributed on the array. To minimise batch variation, each batch was run with equal numbers of $\mathrm{HPV}+, \mathrm{HPV}-$ cancers and controls. Raw data files were imported into Affymetrixs miRNA QCTool and normalised using the quantiles normalisation and median Polish summarisation following a background correction, which corrects for the GC content of each particular probe. Log2 intensities of the 847 human miRNAs were imported into the Data Analysis software package Qlucore Omnics Explorer 2.1 (Qlucore AB 2008-2011, Idéon Science Park, Lund, Sweden). Class comparison analysis was performed using the Student's $t$-test. Comparisons of cancers $v s$ controls were performed for each of the categories. The principal component analysis (PCA) visualisation of the clustering of samples using the genes selected in the class comparison was performed using the build-in PCA tool in Qlucore Omnics Explorer 2.1.

The number of miRNAs found to be differentially expressed in both the HPV + HNSCC tissues and in the cervical tissue was compared with the number of miRNAs differentially expressed in both the HPV - HNSCC tissues and in the cervical tissue. To assess if the difference was significant, a permutation test was developed using the software R (R Development Core Team, 2010).

\section{qPCR}

qPCR validation was carried out on miRNAs selected from the miRNA microarray analysis. cDNA was prepared from 50 to $100 \mathrm{ng}$ total RNA from fresh frozen samples and FFPE samples using the TaqMan MicroRNA Reverse Transcription Kit (Applied Biosystems, Life Technologies Corporation, Carlsbad, CA, USA) and applied to TaqMan MicroRNA Assays containing predesigned primers for mir-16b, mir-363, mir-145, mir-497, mir-381, mir-5743p, mir-20b and mir-15a. Hsa-mir-191 and RNU-44 were used for endogenous control. Quantitative RT-PCR reaction was performed using the TaqMan Universal PCR Master Mix No AmpEras UNG, according to the manufactures instructions, all from Applied Biosystems (Life Technologies Corporation). Each amplification reaction was performed in triplicate, and median value of the three-cycle threshold was used for further analysis. For calculations of fold changes we used the $2^{-\Delta \mathrm{Ct}}$ method.

\section{RESULTS}

\section{Patient characteristics}

Patient characteristics for the tonsillar patients are summarised in Table 1. It was not possible to obtain information about one HPV - TSCC patient because the patient was from Greenland and did not have a Danish chart. The mean age of all TSCC patients was 55. The HPV + cancer patient group and the control group were 8

Table I Patient characteristics in laser microdissected FFPE tonsillar samples

\begin{tabular}{|c|c|c|c|}
\hline & $\begin{array}{l}\text { HPV positive } \\
\text { TSCC }\end{array}$ & $\begin{array}{l}\text { HPV negative } \\
\text { TSCC }\end{array}$ & Controls \\
\hline \multicolumn{4}{|l|}{ Sex } \\
\hline Male & 9 & 10 & 6 \\
\hline Female & I & & 4 \\
\hline Age (Mean) & 56 & 63 & 46 \\
\hline \multicolumn{4}{|l|}{ Stage } \\
\hline II & & 1 & \\
\hline III & 2 & & \\
\hline IVA & 4 & 7 & \\
\hline IVB & 3 & I & \\
\hline IVC & 1 & & \\
\hline Unknown & & I & \\
\hline \multicolumn{4}{|l|}{ Differentiation } \\
\hline Poorly & 6 & 5 & \\
\hline Intermediate & 4 & 4 & \\
\hline Unknown & & I & \\
\hline \multicolumn{4}{|l|}{ Alcohol abuse } \\
\hline Yes & 4 & 2 & I \\
\hline No & 6 & 6 & 8 \\
\hline Unknown & & 2 & I \\
\hline \multicolumn{4}{|l|}{ Tobacco abuse } \\
\hline Current & 3 & 8 & 5 \\
\hline Former & 4 & I & \\
\hline Never & 3 & i & 5 \\
\hline \multicolumn{4}{|l|}{ P16 } \\
\hline Positive & 10 & 3 & \\
\hline Negative & 0 & 7 & \\
\hline \multicolumn{4}{|l|}{$P C R$} \\
\hline Positive & 10 & 0 & I \\
\hline Negative & 0 & 10 & 9 \\
\hline
\end{tabular}

Abbreviations: $F F P E=$ formalin fixed paraffin embedded; HPV = human papilloma virus; TSCC $=$ tonsillar squamous cell carcinoma. 
Table 2 A total of 36 differentially expressed miRNAs comparing HPV + TSCC and HPV - TSCC $(P<0.01, q<0.15$ and fold changes $>1.5)$

\begin{tabular}{|c|c|c|c|}
\hline HPV + /HPV - & $P$-value & $q-$ Value & Fold change \\
\hline hsa-miR-363_st & $1.82 E-05$ & $0.0077|224|$ & 10 \\
\hline hsa-miR-2I_st & 0.001653245 & 0.063649926 & 5.3 \\
\hline hsa-miR-I50_st & 0.002013624 & 0.068221594 & 3.8 \\
\hline hsa-miR-146b-5p st & 0.003291349 & 0.092955818 & 3.5 \\
\hline hsa-miR-15a_st & 0.004978216 & 0.117126349 & 3.4 \\
\hline hsa-miR-20b st & 0.006161643 & 0.13381825 & 3.1 \\
\hline hsa-miR-I46a_st & 0.003292414 & 0.092955818 & 3 \\
\hline hsa-let-7g_st & 0.000378428 & 0.028654156 & 2.9 \\
\hline hsa-miR-625 st & 0.005353066 & 0.122541819 & 2.8 \\
\hline hsa-miR-155_st & 0.00081144 & 0.036173155 & 2.6 \\
\hline hsa-miR-I5b_st & 0.000624782 & 0.031128835 & 2.6 \\
\hline hsa-miR-29a_st & 0.000542066 & 0.0302208 & 2.6 \\
\hline hsa-let-7f st & 0.000405476 & 0.028654156 & 2.6 \\
\hline hsa-miR-125b_st & 0.000519276 & 0.0302208 & 2.2 \\
\hline hsa-miR-26b st & 0.003800803 & 0.100602505 & 2 \\
\hline hsa-miR-342-3p_st & $0.00475 \mid 18$ & 0.114978563 & 2 \\
\hline hsa-miR-16_st & 0.000101751 & 0.012311862 & 1.9 \\
\hline hsa-miR-768-3p_st & 0.002282882 & 0.074369259 & 1.8 \\
\hline hsa-miR-34a-star_st & 0.006431692 & 0.136191078 & 1.7 \\
\hline hsa-miR-596 st - & 0.000405962 & 0.028654156 & 1.6 \\
\hline hsa-miR-20b-star st & 0.005589569 & 0.124588547 & 1.5 \\
\hline hsa-miR-598_st & $6.79 E-05$ & 0.009661928 & 1.5 \\
\hline hsa-miR-200c st & 0.001818879 & 0.066982197 & -1.6 \\
\hline hsa-let-7e_st & 0.004208445 & 0.104839803 & -1.9 \\
\hline hsa-miR-1275 st & $6.01 E-05$ & 0.009661928 & -2.0 \\
\hline hsa-miR-181b st & 0.002641257 & 0.079898032 & -2.0 \\
\hline hsa-miR-324-5p_st & $6.84 \mathrm{E}-05$ & 0.009661928 & -2.1 \\
\hline hsa-miR-423-5p_st & 0.001932585 & $0.068204 \mid 37$ & -2.1 \\
\hline hsa-miR-125a-3p_st & $3.03 E-05$ & 0.008556386 & -2.2 \\
\hline hsa-miR-99b st & 0.001131592 & 0.045640877 & -2.4 \\
\hline hsa-miR-744_st & 0.00021728 & 0.023004549 & -2.7 \\
\hline hsa-miR-877_st & 0.000757677 & 0.035652894 & -2.8 \\
\hline hsa-miR-99b-star st & $7.45 E-06$ & 0.006308449 & -3.0 \\
\hline hsa-miR-I I 80 st & 0.000541339 & 0.0302208 & -3.4 \\
\hline hsa-miR-31 st & 0.003962398 & 0.101701547 & -4.7 \\
\hline hsa-miR-193b-star_st & $0.00096729 \mid$ & 0.040964776 & -5.1 \\
\hline
\end{tabular}

Abbreviations: $\mathrm{HPV}=$ human papilloma virus; $\mathrm{miRNA}=$ microRNA; $\mathrm{TSCC}=$ tonsillar squamous cell carcinoma.

and 17 years (mean) younger than the HPV - cancer group, respectively. Moreover, there was a higher proportion of females in the control group compared with the cancer groups. Only one female was present in the cancer groups presenting with a HPV+ cancer.

The mean age for the CSCC patients was 48 years whereas for the cervical controls it was 43 . Cancer stages were distributed with two patients having stage $\mathrm{Ib}$, one patient stage IIa, two patients stage IIb and five patients stage IIIb. Tumour differentiation was three patients having intermediate differentiation, four patients having low differentiation and three patients were unreported.

\section{microRNA expression in FFPE tonsillar specimens}

To obtain an overview of the differences and similarities of the miRNA expression among the HPV $+I-$ TSCC and normal tissues groups, we first compared the miRNA expression of all samples in a PCA including all variables (Figure 1). In the uncensored PCA plot, samples grouped clearly into three distinct groups, demonstrating that miRNA signatures can distinguish normal tonsillar mucosa from the malignant tumours. Furthermore, miRNA signatures can also separate HPV + tumours from HPVtumours. In a multigroup comparison analysis with a significance level of $P<0.01$ (false discovery rate $(q)<0.06$ ) - 144 miRNAs were differentially expressed between the three groups (Figure 1).

As the HPV + and HPV - tumours were clearly different, we subsequently examined $\mathrm{HPV}+$ and $\mathrm{HPV}-$ tumour samples separately and compared them with the normal samples in a censored class comparison analysis. Using a significance level of $P$-values $<0.01 \quad(q<0.05)$, we found 116 miRNAs to be differentially expressed between HPV + TSCC and controls. A total of 111 miRNAs were differentially expressed between HPV - TSCC and controls. In a comparison of HPV + TSCC to HPV - TSCC, we found 36 differentially expressed miRNAs $(P$-value $<0.01, q$-value $<0.15$ and fold changes $>1.5)$. In all, 22 miRNAs were upregulated and 14 were downregulated (Table 2).

\section{microRNA expression in cervical specimens}

Differentially expressed miRNAs from the cervical specimens were visualised in a PCA plot, including all variables, and it was noticed that the CSCC samples and control samples clustered into two distinct groups, demonstrating that the miRNA expression differentiates normal cervical tissue and CSCC (Figure 2). In a censored two group class comparison analysis, using a significance level of $P$-values $<0.01(q<0.05)$, 148 miRNAs were differentially expressed between the CSCC and cervical controls (Figure 2). As CSCC is known to be driven by HPV, we used the 148 miRNAs differentially expressed in CSCC as a reference for HPV-mediated changes (Supplementary Table 1).

Eight miRNAs were selected for validation by qPCR. MiR-363, miR-16, mir-574-3p, mir-20b and mir-15a were used for the FFPE samples. Whereas the fresh frozen samples (HPV + PSCC, HPV oral SCC (OSCC), pharyngeal/oral controls and all cervical SCC and controls) were additionally tested for mir-145, miR-497 and mir381. In each case, cancer $v s$ controls was compared. The results of the qPCR validation are shown in Supplementary Figure 2. By qPCR miR-363 was also found upregulated in the CSCC samples and miR497 was also found downregulated by qPCR in the HPV - PSCC. In all other cases qPCR results confirmed the array data.

\section{Comparison of all HPV + and HPV - HNSCCs with CSCC}

In an attempt to define common HPV-controlled miRNAs, that are independent from tissue type and anatomical localisation, we compared the differentially expressed miRNAs of HPV + / HPV HNSCC and their respective controls and compared them to the miRNAs in the HPV + CSCCs. The list of differentially expressed miRNAs from the HPV+ FFPE TSCC was merged with our previously published differentially expressed miRNAs from fresh frozen HPV + PSCC and defined as HPV + HNSCC miRNAs. In this group, we identified 144 differentially expressed miRNAs. Likewise, the list of differentially expressed miRNAs of HPV FFPE TSCC was merged with previously published differentially expressed miRNAs in fresh frozen HPV - PSCC and defined as HPV - HNSCC miRNAs. Here, we identified 140 differentially expressed miRNAs. Subsequently we compared the miRNA expression profiles in the HPV + and HPV - HNSCC group with the miRNA expression profiles in CSCC.

How these two sets of miRNAs overlap with each other and the miRNAs identified in the cervical samples is illustrated in the Venn diagram (Figure 3).

Of particular interest is the number of miRNAs shared exclusively by HPV + HNSCC and CSCC compared with the number of miRNAs shared exclusively by HPV - HNSCC and CSCC. As seen from the diagram $d=n_{+}-n_{-}=23-6=17$. The distribution of $d$ under the null hypothesis that HPV + and HPV - samples are exchangeable is shown in Supplementary Figure 3. In 4 out of 1000 permutations, the value of $d$ turned out $\geq 17$, giving the test a $P$-value of 0.004 . The permutation test was also run with a harder cutoff (false discovery rate $q<0.05$ by the Benjamin-Hochberg adjustment for multiple testing) with a similar result $(P<0.032)$.

To be absolutely certain of identifying miRNAs that are exclusively present in HPV + tumours, additionally 7 miRNAs 
A

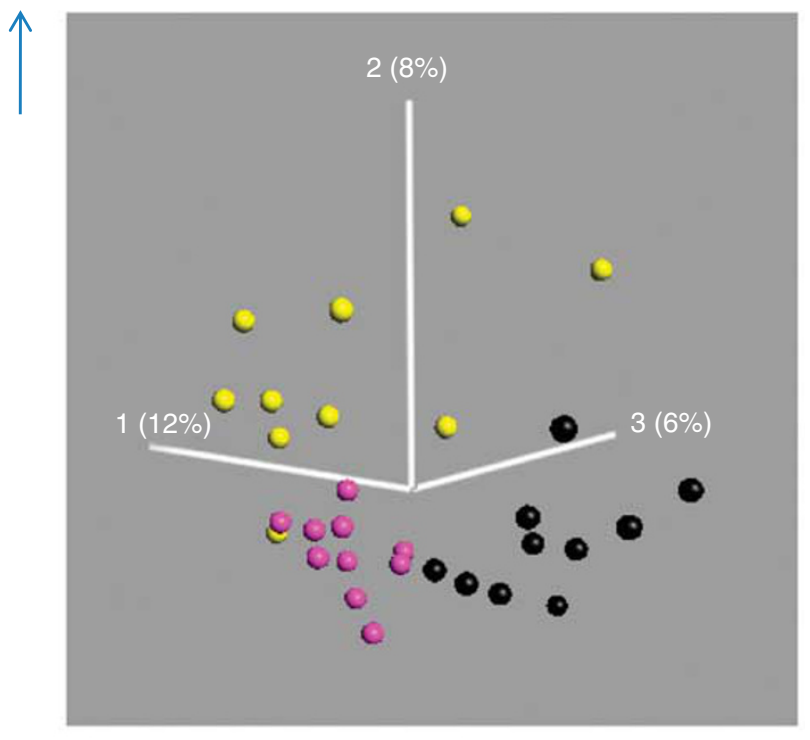

B

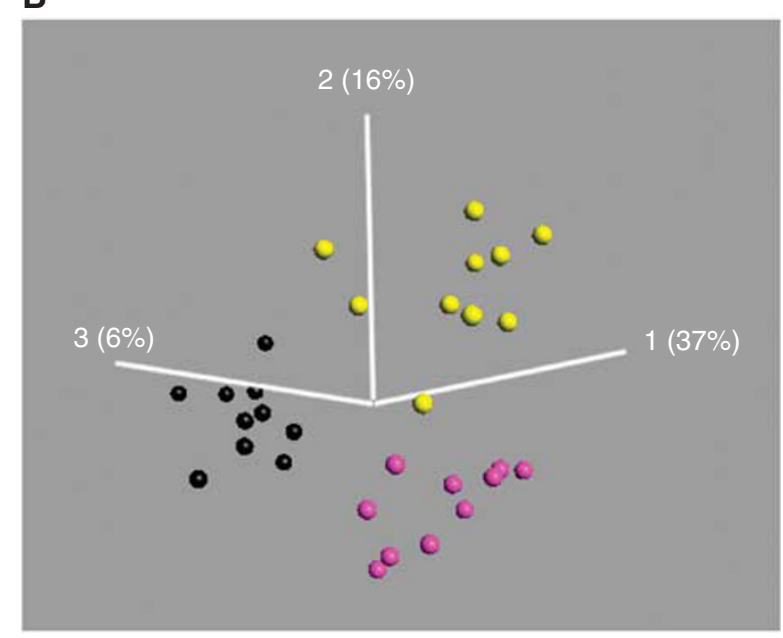

C

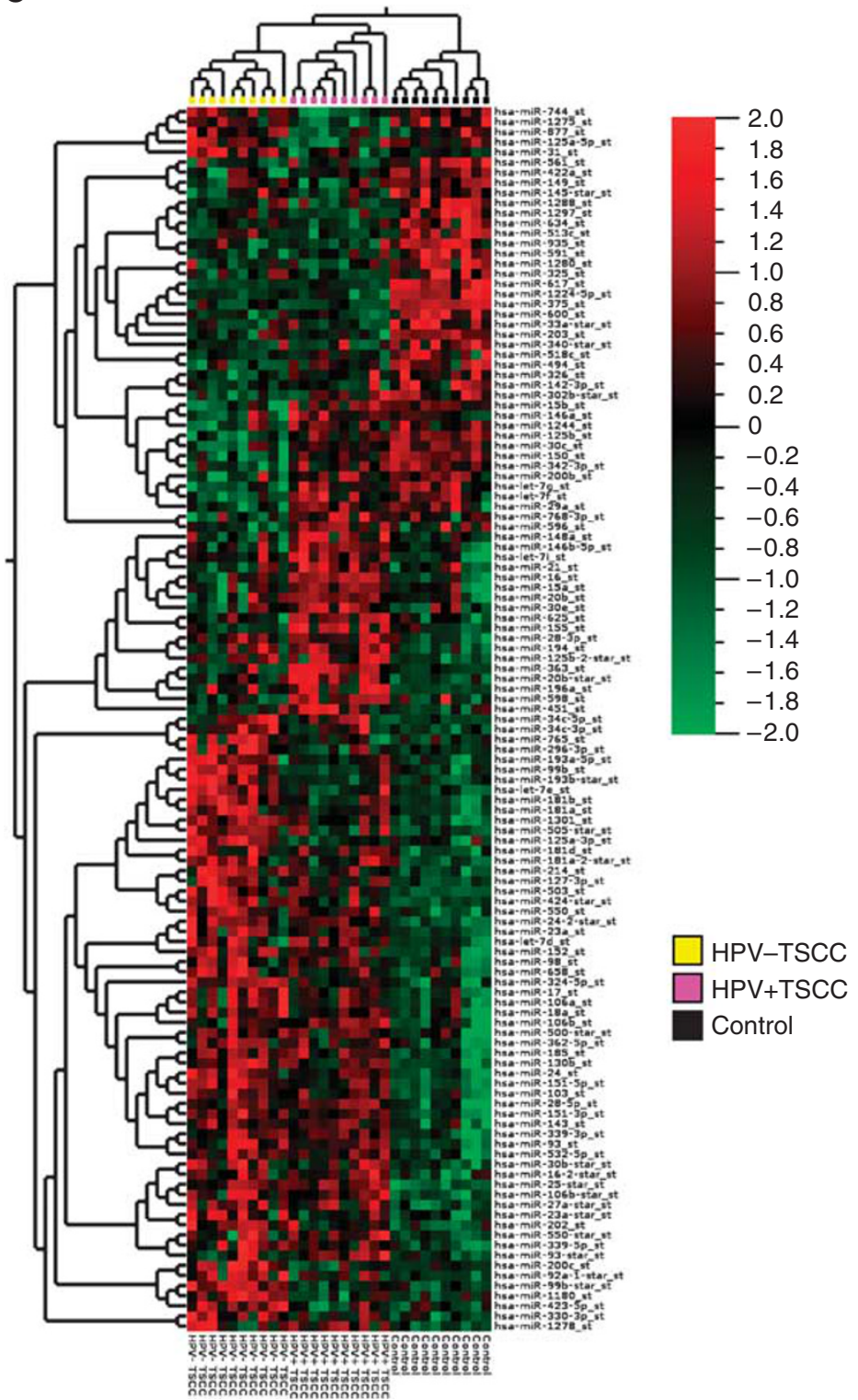

Figure I (A) Principal component analysis (PCA) plot including all variables. Human papilloma virus - TSCC (yellow), HPV + TSCC (purple) and tonsillar controls (black). (B) Principal component analysis plot, visualising differentially expressed miRNAs in HPV +, HPV - and tonsillar control, subset of variables. $(P<0.01, q<0.06)$. (C) Heat map: three distinct groups were observed. Human papilloma virus - TSCC (yellow), HPV + TSCC (purple) and tonsillar controls (black). The heatmap and PCA visualisation was performed using the Qlucore Gene Expression Explorer (http://www.qlucore.com).

were excluded from the 23 miRNAs identified only in HPV+ HNSCC and CSCC, as they have previously been identified in HPV - OSCCs by our study group (Lajer et al, 2011). microRNAs denoted with a star was likewise excluded. That leaves 14 miRNAs to be defined as HPV core miRNAs, which are listed in Table 3. In all, 4 were upregulated and 10 were downregulated. The upregulated HPV core miRNAs were miR-10b, miR-15a, mir-20b and miR-16 and among the downregulated HPV core miRNAs there were miR-139-3p, miR-145, miR-381 and miR-574-3p.

microRNAs that were common for all SCC independent of HPV status and compared with their respective normal tissue could therefore be defined as SCC cancer-specific miRNA. These are listed in Table 4 and include the miRNAs that were identified in $\mathrm{HPV}+\mathrm{HNSCC} / \mathrm{HPV}-\mathrm{HNSCC}$ and CSCC as well as previously identified in OSCC (Lajer et al, 2011). Finally, 45 (from the Venn Diagram) miRNAs were common between the HPV + HNSCC and HPV - HNSCC but not differentially expressed in the CSCC. These
miRNAs seem to be cancer-specific miRNAs that are dependent on an anatomical localisation within the head and neck.

\section{DISCUSSION}

The presence of HPV defines a distinct group of head and neck cancers exhibiting epidemiological, histopathological, clinical and prognostic differences compared with the typical HNSCC. Although HPV is acknowledged as the cause of the vast majority of cervical cancers, it is still debated which role HPV has in HNSCC cancers. To exploit the role of HPV in HNSCC, it is important to characterise the molecular basis of $\mathrm{HPV}+$ and HPV - HNSCC which can be further strengthened by comparison with the carcinogenic mechanisms to HPV + CSCC.

This study is the first to make a direct correlation of miRNA expression in HPV + HNSCC to CSCC in the same settings and in 
A

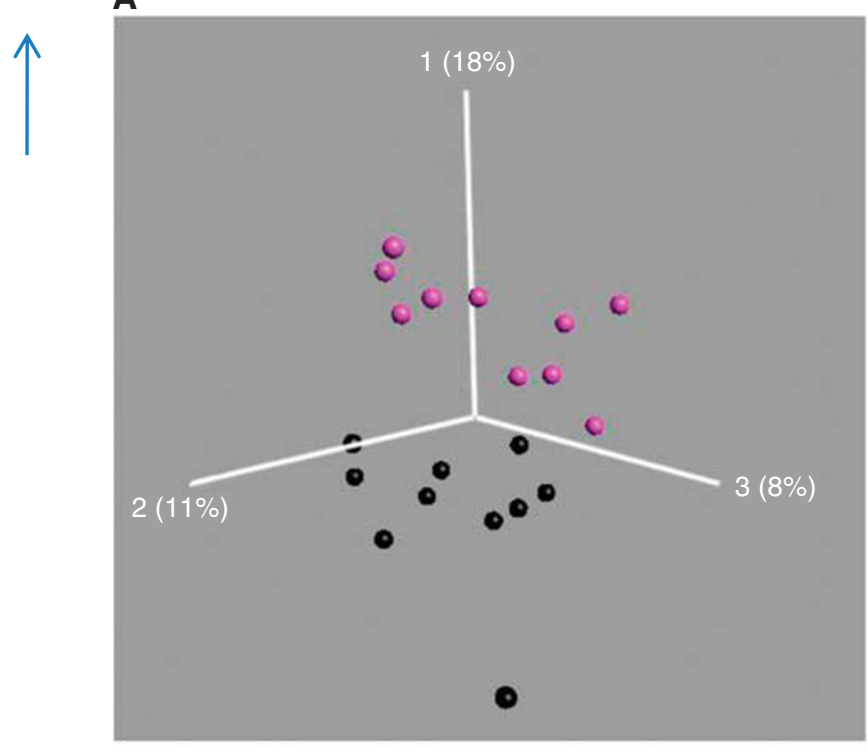

B

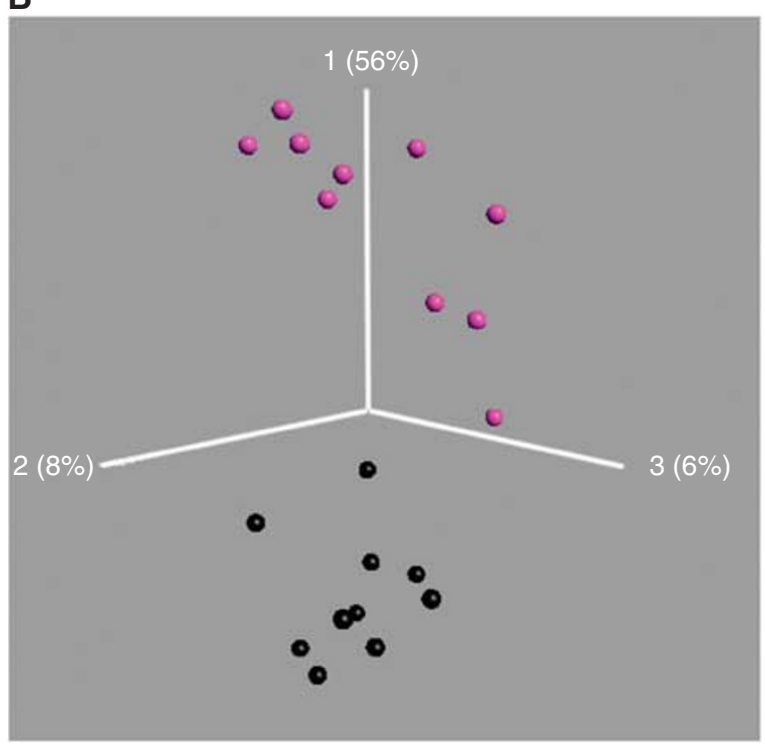

C

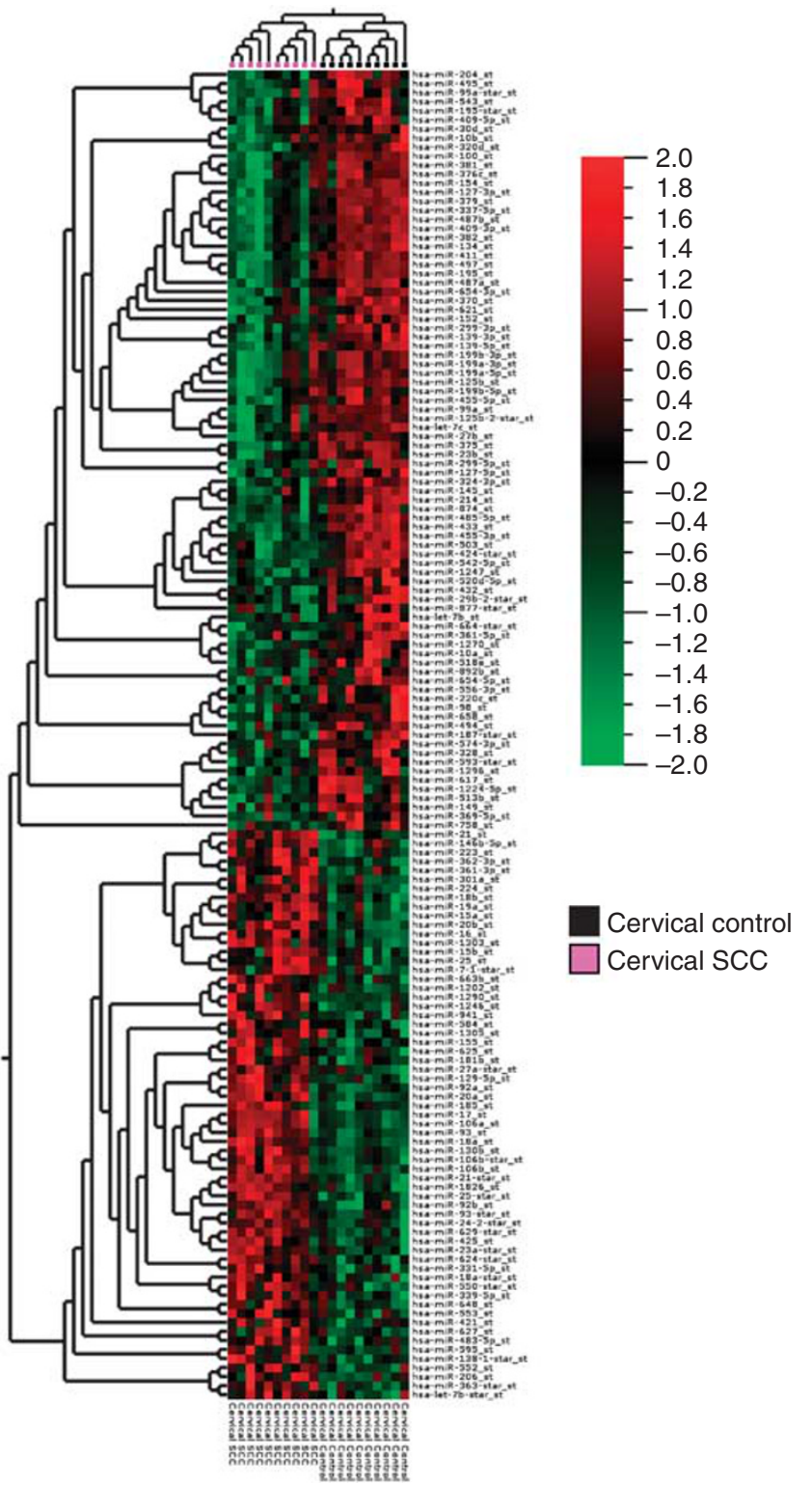

Figure 2 (A) Principal component analysis plot including all variables. (B) Principal component analysis plot, visualising differentially expressed miRNAs in CSCC compared with cervical controls. (C) Heat map $(P<0.01, q<0.05)$. Cervical squamous cell carcinoma (CSCC) (purple), cervical controls (black).

that way, it demonstrates the impact of HPV on the miRNA expression in solid malignant tumours irrespective of the anatomical site.

The demonstration of HPV-dependent perturbations on miRNA expression adds to the complexity of HPV-induced transformation. Human papilloma virus elicits widespread changes in cellular gene expression, which may at least partially be responsible for the distinct clinical behaviour of HPV + cancers.

In this study, we show that HPV+ HNSCC have a distinct miRNA profile that clearly distinguishes them from HPVHNSCC. Furthermore, there are several similarities in the miRNA expressions of SCC in spite of two different anatomical localisations. microRNAs are highly tissue specific (Rosenfeld et al, 2008; Rosenwald et al, 2010). As expected we found a high degree of concordance between HPV + and HPV - HNSCC and a much lesser concordance between CSCC and HNSCC. Interestingly, however, the permutation test confirmed that HPV + HNSCC and $\mathrm{HPV}+\mathrm{CSCC}$ were significantly $(P=0.004)$ more alike compared with HPV - HNSCC and CSSC.
It was striking that the miRNAs identified as HPV core miRNAs appear to involve closely related miRNAs united in a few clusters and miR-families and they have distinct interactions within theE6 p53 and E7/pRb pathways of HPV. Figure 4 illustrates the proposed interactions of the HPV core miRNAs in the HPVinduced malignant pathogenesis.

Among the HPV core miRNAs, we identified five members of the miR-family 'miR-15a, miR-16, miR195, miR-424 and miR-497'. We observed increased levels of miR-15a and miR-16 and decreased levels of miR-195 and miR-497 (the latter two miRNAs were also significantly reduced in HPV - HNSCC although to a lesser degree). These miRNAs target mRNAs-encoding proteins involved in G1/S checkpoint and they have been considered as tumour suppressors. Studies show that cell lines lacking functional $\mathrm{Rb}$ (as in HPV-driven cancer) are resistant to mir-15a-16 cell cycle arrest and reintroduction of functional $\mathrm{Rb}$ resensitises the cells (Bandi et al, 2009). Mir-15a and mir-16 are often downregulated in cancer and their expression is inversely correlated with the 


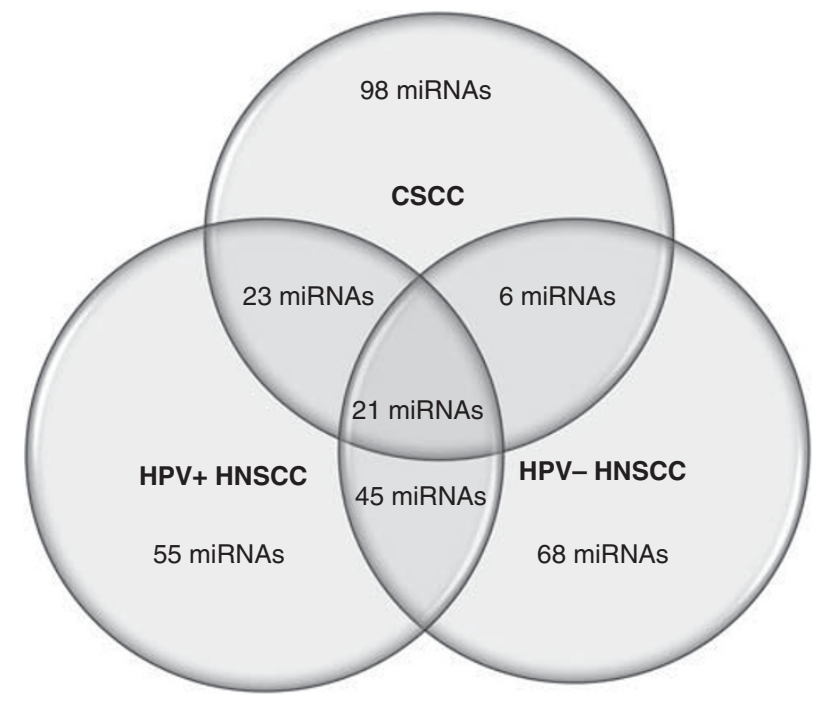

Figure 3 Venn diagram illustrating the number of miRNAs differentially expressed in the three groups (HPV + HNSCC, HPV - HNSCC and CSCC) compared with their normal counterpart and how they overlap with each other $(P<0.0 \mathrm{I})$.

Table 3 Human papilloma virus core miRNAs

\begin{tabular}{lllll}
\hline Probeset name & $\begin{array}{c}\text { P-value } \\
\text { CSCC }\end{array}$ & $\begin{array}{c}\text { Fold } \\
\text { change } \\
\text { CSCC }\end{array}$ & $\begin{array}{c}\text { P-value } \\
\text { HPV }+ \\
\text { HNSCC }\end{array}$ & $\begin{array}{c}\text { Fold } \\
\text { change } \\
\text { HPV }+ \\
\text { HNSCC }\end{array}$ \\
\hline hsa-miR-10b_st & 0.003372 & 2.12 & 0.001619 & 1.79 \\
hsa-miR-15a_st & 0.0026574 & 2.02 & 0.00155905 & 4.19 \\
hsa-miR-16_st & $1.01 E-05$ & 1.86 & 0.00000042 & 2.40 \\
hsa-miR-20b_st & 0.000273 & 2.19 & 0.000873978 & 3.42 \\
hsa-miR-139-3p_st & 0.0005829 & -3.03 & 0.00114667 & -2.26 \\
hsa-miR-139-5p_st & 0.0001662 & -3.33 & $0.0051409 \mid$ & -1.74 \\
hsa-miR-145_st & 0.0032745 & -2.17 & 0.00006312 & -2.19 \\
hsa-miR-199a-3p_st & 0.003554 & -2.36 & 0.009824 & -1.65 \\
hsa-miR-199a-5p_st & 0.000938 & -2.66 & 0.006027 & -1.75 \\
hsa-miR-199b-5p_st & 0.0028727 & -4.53 & 0.00007410 & -1.85 \\
hsa-miR-328_st & 0.0076266 & -1.81 & 0.00584300 & -1.81 \\
hsa-miR-379_st & $1.29 E-06$ & -8.29 & 0.00568124 & -1.5 \\
hsa-miR-38I_st & 0.0014045 & -4.22 & 0.00612568 & -1.46 \\
hsa-miR-574-3p_st & 0.0008629 & -2 & 0.00909130 & -1.62 \\
\hline
\end{tabular}

Abbreviations: $\mathrm{CSCC}=$ cervical squamous cell carcinoma; $\mathrm{HNSCC}=$ head and neck SCC; HPV = human papilloma virus; miRNA = microRNA; OSCC $=$ oral squamous cell carcinoma; TSCC $=$ tonsillar SCC. $P<0.01$. MiRNAs with star or formerly shown to be present in OSCC is removed.

expression of cyclin D1. In non-small cell lung cancer cell lines the cyclines D1, D2 and E1 have been shown to be directly regulated by the level of mir-15a/mir16 and overexpression of these induces cell cycle arrest in the $\mathrm{G}_{1}-\mathrm{G}_{0}$ phase.

The most markedly and consistently upregulated miRNA in HPV + TSCC was miR-363 (Supplementary Table 3). This is in concordance with both our previous study and the study by (Wald et al, 2011), demonstrating a considerable HPV 16 E6-dependent upregulation of miR-363 in HPV + cell lines. In agreement with other studies (Wang et al, 2008; Pereira et al, 2010), miR-363 was not found differentially expressed in our CSCC specimens in the array data, though it was upregulated by qPCR. The miR-106-363 cluster was recently reported implicated in T-cell leukaemia (Landais et al, 2007), and overexpression of the miR-106-363 cluster induced anchorage independent growth of NIH/3T3 cells. Five of the six miRNAs in this cluster were upregulated in the CSCC samples (miR-106a, miR18b, miR-20b miR92a and miR-92b;
Table 4 Cancer-specific miRNAs, that is, differentially expressed miRNAs in cancer vs controls in all three categories (HPV + HNSCC (FFPE TSCC/FF PSCC), HPV - HNSCC (FF PSCC/FFPE TSCC) and CSCC

\begin{tabular}{|c|c|c|c|c|}
\hline Probeset name & $P$-value & $\begin{array}{l}\text { Fold } \\
\text { change } \\
\text { CSCC }\end{array}$ & $P$-value & $\begin{array}{c}\begin{array}{c}\text { Fold } \\
\text { change }\end{array} \\
\text { HNSCC } \\
(H P V+ \\
\text { or HPV-) }\end{array}$ \\
\hline hsa-miR-17_st & $9.22 E-05$ & 1.81 & 0.00442863 & 1.63 \\
\hline hsa-miR-2I_st \# & $4.99 E-06$ & 3.36 & 0.00437099 & 5.16 \\
\hline hsa-miR-93_st & 0.0001176 & 2.07 & $4.69 E-06$ & 2.20 \\
\hline hsa-miR-99a_st \# & 0.000337 & -5.47 & 0.00715179 & -1.84 \\
\hline hsa-miR-100_st & $6.00 E-06$ & -5.25 & $1.12 E-05$ & -2.76 \\
\hline hsa-miR-106a_st & $7.69 E-05$ & 1.80 & 0.00405 & 1.95 \\
\hline hsa-miR-106b_st & 3.10E-05 & 1.70 & $6.39 E-05$ & 4.27 \\
\hline hsa-miR-125b_st & 0.000171 & -2.56 & $9.12 \mathrm{E}-07$ & -2.13 \\
\hline hsa-miR-130b_st & 0.0004062 & 2.61 & $5.27 \mathrm{E}-06$ & 1.98 \\
\hline hsa-miR-1 46b-5p st \# & 0.0025381 & 2.44 & 0.00032341 & 4.72 \\
\hline hsa-miR-149_st ${ }^{\#-}$ & 0.0084416 & -3.16 & 0.00323463 & -4.18 \\
\hline hsa-miR-155_st ${ }^{\#}$ & 0.0004656 & 2.93 & 0.00217636 & 2.46 \\
\hline hsa-miR-185_st & $0.00439 \mid$ & 1.65 & 0.00010159 & 2.46 \\
\hline hsa-miR-195_st & $2.15 E-06$ & -5.60 & 0.000227019 & -2.00 \\
\hline hsa-miR-339-5p_st & $0.0077 \mid 82$ & 1.59 & 0.0005106 & 1.77 \\
\hline hsa-miR-375_st & $7.44 \mathrm{E}-07$ & -33.33 & $6.33 E-12$ & -90.00 \\
\hline hsa-miR-497_st & $2.68 \mathrm{E}-07$ & -7.33 & $4.56 \mathrm{E}-05$ & -2.63 \\
\hline hsa-miR-617 st & 0.0023253 & -6.25 & $8.99 E-08$ & -14.29 \\
\hline hsa-miR-625_st ${ }^{\#}$ & 0.0059367 & 2.08 & 0.001111112 & 3.38 \\
\hline hsa-miR-94l st \# & 0.0020493 & 2.30 & 0.00512539 & 1.65 \\
\hline hsa-miR-1224-5p_st & 0.004525 & 3.74 & $2.95 E-06$ & 14.97 \\
\hline
\end{tabular}

Abbreviations: $C S C C=$ cervical squamous cell carcinoma; FFPE $=$ formalin fixed paraffin embedded; HNSCC = head and neck SCC; HPV = human papilloma virus; miRNA = microRNA; OSCC $=$ oral squamous cell carcinoma; $P S C C=$ pharyngeal SCC; TSCC = tonsillar SCC. "Formerly identified in HPV - OSCC.

Supplementary Table 1). Besides miR-363, also miR-20b was upregulated in the HPV + HNSCC.

We identified miR-145 in the core set of HPV-controlled miRNAs as it was downregulated in CSCC as well as in HPV+ HNSCC. MiR-143 was also downregulated in both HPV + HNSCC and in CSCC, but did not reach a significance level of $P<0.01$ $(P=0.011)$ in the cervical samples. MiR-143 and miR-145 have previously been shown to suppress cell growth in CSCC and they have consequently been implicated in cervical carcinogenesis (Wang et al, 2008). Repression of miR-143-145 could be the result of the HPV oncogene E6 as shown in Figure 4. Even though a reduction of miR-143 and miR-145 seems to be related to HPV in head and neck cancers, reduced expression of these miRNAs have been described in other tumour types unrelated to HPV presence (Michael et al, 2003; Akao et al, 2007a, b).

Reduced levels of miR-195, miR-143 and miR-145 may be secondary to E6-dependent loss of p53/p63/p73 that normally regulate these miRNAs (Boominathan, 2010). Zheng and Wang (2011)also describes how the viral oncoproteins E6 and E7 deregulate the expression of the miR-15/16 cluster, the miR-17-92 family and miR-106b/93/25 cluster via the E6-p53 and E7-pRb pathways in HPV cervical cancers (Zheng and Wang, 2011). This study supports these findings in HPV + HNSCC as well.

Although HPV + and HPV - tumours could be clearly distinguished, the differences in miRNA expression were diminutive. In general, the presence of HPV DNA in the cancer samples was associated with downregulation of miRNAs in CSCC and HPV + HNSCC (10 of 14, Table 4).

A difference in gene expression profiles between $\mathrm{HPV}+$ and HPV - HNSCC as well as a resemblance of the HPV + HNSCC with CSCC have been reported in another study (Pyeon et al, 2007). We can now deduce that both miRNA and mRNA expression profiles clearly display differences in HPV + and HPV- HNSCC. In addition, the miRNA and mRNA expression in HPV+ HNSCC 


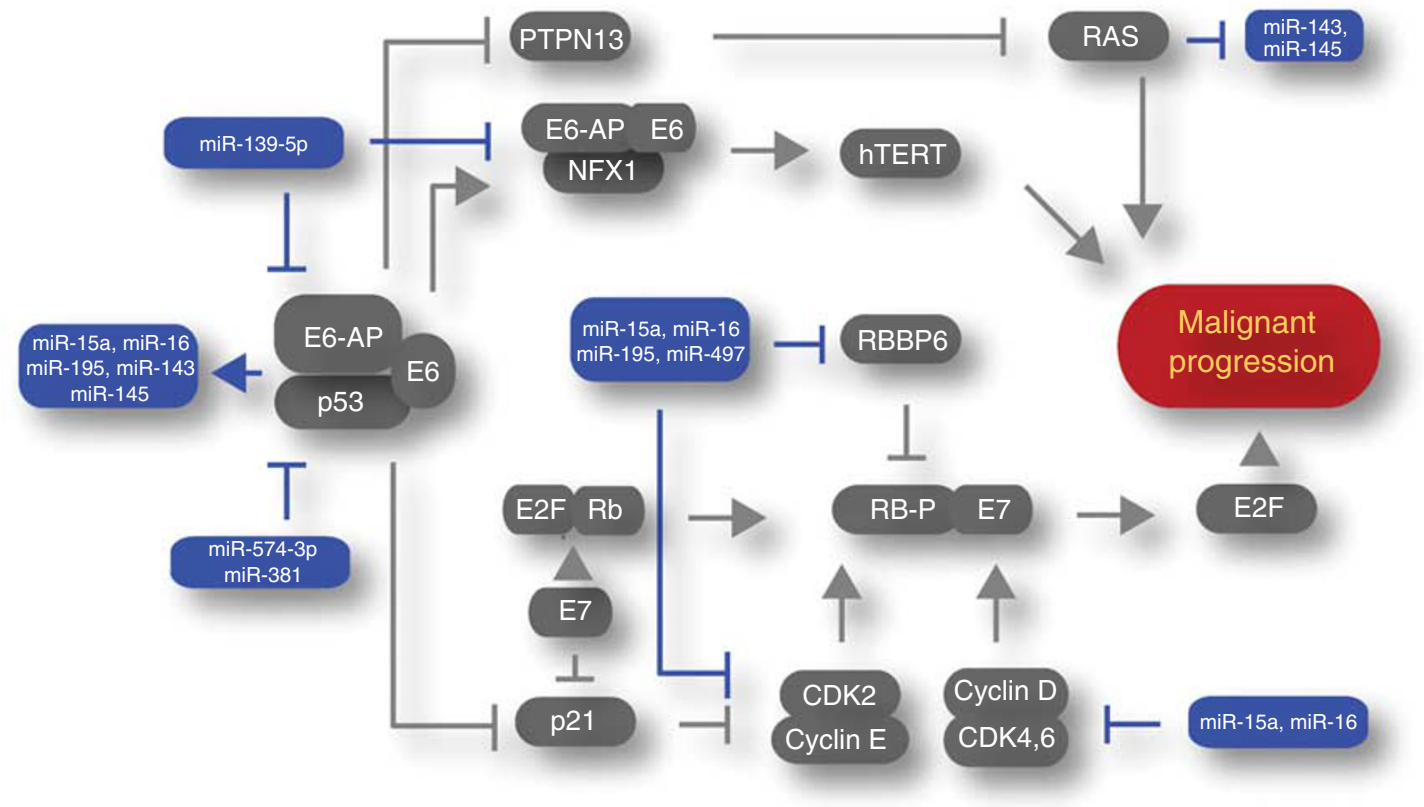

Figure 4 The schematic representation of the relation between HPV-regulated miRNAs and factors involved in malignant transformation caused by the viral oncoproteins E6 and E7. Putative miRNA-regulated proteins and miRNA controlled by transforming factors are indicated in grey and miRNAs are shown in blue boxes. Binding of E7 to $\mathrm{pRb}$ leads to degradation of pRb and release of transcription factor E2F, which drives the cell into S-phase and cell division. Interaction between E7 and p2I results in inhibition of the cyclins, which are targeted by miR-15, miR-16, miR-195, miR-497, and this leads to inhibition of cell cycle arrest and contribute to carcinogenesis. Ubiquitination by E6 and ubiquitin ligase E6AP (potentially targeted by miR- I39-5p) leads to p53 degradation and subsequently impaired apoptosis following DNA damage. p53 is also potentially targeted by miR-574-5p and miR-38I, and p53 in turns regulates transcription of miR-15, miR-16, miR-143, miR-145 and miR-195 By association with E6/E6AP, NFXI is degraded and results in activation of hTERT, which leades to cellular immortalisation. E6 degrades the tumour supressor PTPNI3 and loss of PTPNI3 further enhances the oncogenic Ras signalling. Ras further regulates miR-I43 and miR-I45 transcription by binding to the promoter.

exhibit similarities to the known HPV-induced CSCC. This further elucigcqias the oncogenic role of HPV in different anatomical sites.

This study reflects the global change in miRNA expression of the different cancer types. The intention is, as suggested by Gallagher et al (2010), to investigate the collective changes in many miRNAs (global profiling), which may be the most interesting biologically parameter to consider - compared with choosing one miRNA for further functional analysis in a cancer cell line, which may be far from the conditions in vivo. It is most likely that miRNAs work cooperatively in vivo and that physiological regulation of a single protein by a single miRNA may be a rather rare occurrence. In accordance with this, we observed that changes of miRNA expression often implicated a cluster of miRNAs or a whole miRNA family.

Most of the HPV core miRNAs identified in this study have not been investigated in relation to HNSCC, as most miRNA studies regarding HNSCC have not taken the HPV status into consideration. Further functional studies of these miRNAs in HPV + and HPV - HNSCC cell lines will be needed in the future.

It is the combination of the HPV core miRNAs that seems to be closely related to HPV infection, regardless of the fact that the individual miRNAs may not be unique to HPV-driven cancer. Consequently, some of the HPV core miRNAs have also been identified in other types of HPV - cancers. This aspect will need further investigations.

\section{REFERENCES}

Akao Y, Nakagawa Y, Kitade Y, Kinoshita T, Naoe T (2007a) Downregulation of microRNAs-143 and -145 in B-cell malignancies. Cancer Sci 98(12): 1914-1920

Akao Y, Nakagawa Y, Naoe T (2007b) MicroRNA-143 and -145 in colon cancer. DNA Cell Biol 26(5): 311-320

\section{CONCLUSION}

In this study, we show that HPV + HNSCC has a distinct miRNA profile compared with HPV - HNSCC. Furthermore, miRNA profiles in HPV + HNSCC are significantly more similar to miRNA profiles of CSCC than HPV - HNSCC. A set of HPV core miRNAs were identified. Of these especially the miR-15a/miR-16/, miR-143/miR-145 and the miR-106-363 cluster appear to have targets within the known HPV pathogenesis. This adds new knowledge to known pathogenic pathways of HPV and substantiates the oncogenic role of HPV in subsets of HNSCCs. The identification of miRNAs associated with different HPV-mediated cancers is important, as they may be useful as general and HPV-specific tumour markers. In addition, they may prove useful in future individualised treatment strategies and development of new targeted therapies.

\section{ACKNOWLEDGEMENTS}

We thank Omid Niazi and Melissa Alexandra Visser for technical assistance. The study was supported by the Oda Pedersens Foundation, Beckett Foundation, Hoerslev Foundation, Aase and Ejnar Danielsen's Foundation and Hans Skouby and Emma Skouby Foundation.

Supplementary Information accompanies the paper on British Journal of Cancer website (http://www.nature.com/bjc)

Bandi N, Zbinden S, Gugger M, Arnold M, Kocher V, Hasan L, Kappeler A, Brunner T, Vassella E (2009) miR-15a and miR-16 are implicated in cell cycle regulation in a $\mathrm{Rb}$-dependent manner and are frequently deleted or down-regulated in non-small cell lung cancer. Cancer Res 69(13): 5553-5559 
Boominathan L (2010) The tumor suppressors p53, p63, and p73 are regulators of microRNA processing complex. PLoS One 5(5): e10615

Gallagher IJ, Scheele C, Keller P, Nielsen AR, Remenyi J, Fischer CP, Roder K, Babraj J, Wahlestedt C, Hutvagner G, Pedersen BK, Timmons JA (2010) Integration of microRNA changes in vivo identifies novel molecular features of muscle insulin resistance in type 2 diabetes. Genome Med 2(2): 9

Ghittoni R, Accardi R, Hasan U, Gheit T, Sylla B, Tommasino M (2010) The biological properties of E6 and E7 oncoproteins from human papillomaviruses. Virus Genes 40(1): 1-13

Hafkamp HC, Manni JJ, Haesevoets A, Voogd AC, Schepers M, Bot FJ, Hopman AH, Ramaekers FC, Speel EJ (2008) Marked differences in survival rate between smokers and nonsmokers with HPV 16-associated tonsillar carcinomas. Int J Cancer 122(12): 2656-2664

Lajer CB, Buchwald CV (2010) The role of human papillomavirus in head and neck cancer. APMIS 118(6-7): 510-519

Lajer CB, Nielsen FC, Friis-Hansen L, Norrild B, Borup R, Garnaes E, Rossing M, Specht L, Therkildsen MH, Nauntofte B, Dabelsteen S, von BC (2011) Different miRNA signatures of oral and pharyngeal squamous cell carcinomas: a prospective translational study. $\mathrm{Br} \mathrm{J}$ Cancer 104(5): $830-840$

Landais S, Landry S, Legault P, Rassart E (2007) Oncogenic potential of the miR-106-363 cluster and its implication in human T-cell leukemia. Cancer Res 67(12): 5699-5707

Lassen P, Eriksen JG, Hamilton-Dutoit S, Tramm T, Alsner J, Overgaard J (2009) Effect of HPV-associated p16INK4A expression on response to radiotherapy and survival in squamous cell carcinoma of the head and neck. J Clin Oncol 27(12): 1992-1998

Majewski S, Bosch FX, Dillner J, Iversen OE, Kjaer SK, Munoz N, Olsson SE, Paavonen J, Sigurdsson K, Bryan J, Esser MT, Giacoletti K, James M, Taddeo F, Vuocolo S, Barr E (2009) The impact of a quadrivalent human papillomavirus (types $6,11,16,18$ ) virus-like particle vaccine in European women aged 16 to 24. J Eur Acad Dermatol Venereol 23(10): 1147-1155

Michael MZ, O'Connor SM, Holst Pellekaan NG, Young GP, James RJ (2003) Reduced accumulation of specific microRNAs in colorectal neoplasia. Mol Cancer Res 1(12): 882-891

Nasman A, Attner P, Hammarstedt L, Du J, Eriksson M, Giraud G, hrlundRichter S, Marklund L, Romanitan M, Lindquist D, Ramqvist T, Lindholm J, Sparen P, Ye W, Dahlstrand H, Munck-Wikland E, Dalianis $\mathrm{T}$ (2009) Incidence of human papillomavirus (HPV) positive tonsillar carcinoma in Stockholm, Sweden: an epidemic of viral-induced carcinoma? Int J Cancer 125(2): 362-366

Pan X, Wang ZX, Wang R (2010) MicroRNA-21: A novel therapeutic target in human cancer. Cancer Biol Ther 10(12): 1224-1232

Pereira PM, Marques JP, Soares AR, Carreto L, Santos MA (2010) MicroRNA expression variability in human cervical tissues. PLOS ONE 5(7): e11780
Pim D, Banks L (2010) Interaction of viral oncoproteins with cellular target molecules: infection with high-risk vs low-risk human papillomaviruses. APMIS 118(6-7): 471-493

Pyeon D, Newton MA, Lambert PF, den Boon JA, Sengupta S, Marsit CJ, Woodworth CD, Connor JP, Haugen TH, Smith EM, Kelsey KT, Turek LP, Ahlquist P (2007) Fundamental differences in cell cycle deregulation in human papillomavirus-positive and human papillomavirus-negative head/neck and cervical cancers. Cancer Res 67(10): 4605-4619

$\mathrm{R}$ Development Core Team (2010) R: a language and environment for statistical computing. R Foundation for Statistical Computing: Vienna, Austria

Rosenfeld N, Aharonov R, Meiri E, Rosenwald S, Spector Y, Zepeniuk M, Benjamin $\mathrm{H}$, Shabes $\mathrm{N}$, Tabak S, Levy A, Lebanony D, Goren $\mathrm{Y}$, Silberschein E, Targan N, Ben-Ari A, Gilad S, Sion-Vardy N, Tobar A, Feinmesser M, Kharenko O, Nativ O, Nass D, Perelman M, Yosepovich A Shalmon B, Polak-Charcon S, Fridman E, Avniel A, Bentwich I, Bentwich Z, Cohen D, Chajut A, Barshack I (2008) MicroRNAs accurately identify cancer tissue origin. Nat Biotechnol 26(4): 462-469

Rosenwald S, Gilad S, Benjamin S, Lebanony D, Dromi N, Faerman A Benjamin H, Tamir R, Ezagouri M, Goren E, Barshack I, Nass D, Tobar A, Feinmesser M, Rosenfeld N, Leizerman I, Ashkenazi K, Spector Y, Chajut A, Aharonov R (2010) Validation of a microRNA-based qRT-PCR test for accurate identification of tumor tissue origin. Mod Pathol 23(6): $814-823$

Syrjanen K, Syrjanen S, Lamberg M, Pyrhonen S, Nuutinen J (1983a) Morphological and immunohistochemical evidence suggesting human papillomavirus (HPV) involvement in oral squamous cell carcinogenesis. Int J Oral Surg 12(6): 418-424

Syrjanen KJ, Syrjanen SM, Lamberg MA, Pyrhonen S (1983b) Human papillomavirus (HPV) involvement in squamous cell lesions of the oral cavity. Proc Finn Dent Soc 79(1): 1-8

Walboomers JM, Jacobs MV, Manos MM, Bosch FX, Kummer JA, Shah KV, Snijders PJ, Peto J, Meijer CJ, Munoz N (1999) Human papillomavirus is a necessary cause of invasive cervical cancer worldwide. J Pathol 189(1): $12-19$

Wald AI, Hoskins EE, Wells SI, Ferris RL, Khan SA (2011) Alteration of microRNA profiles in squamous cell carcinoma of the head and neck cell lines by human papillomavirus. Head Neck 33(4): 504-512

Wang X, Tang S, Le SY, Lu R, Rader JS, Meyers C, Zheng ZM (2008) Aberrant expression of oncogenic and tumor-suppressive microRNAs in cervical cancer is required for cancer cell growth. PLoS One 3(7): e2557

Warnakulasuriya S (2009) Global epidemiology of oral and oropharyngeal cancer. Oral Oncol 45(4-5): 309-316

Wise-Draper TM, Wells SI (2008) Papillomavirus E6 and E7 proteins and their cellular targets. Front Biosci 13: 1003-1017

Zheng ZM, Wang X (2011) Regulation of cellular miRNA expression by human papillomaviruses. Biochim Biophys Acta 1809(11-12): 668-677

This work is published under the standard license to publish agreement. After 12 months the work will become freely available and the license terms will switch to a Creative Commons Attribution-NonCommercial-Share Alike 3.0 Unported License. 